Christiane Eichenberg, Paolo Raile, Stella Becher, Catherine Dapeci, Jessica Pacher, Paul Jo Marc Rach, Birgitta Schiller, Eva Wimmer \& Lisa Winter

Psychothérapie en ligne et télé psychothérapie 


\section{Impressum}

Psychotherapie-Wissenschaft

ISSN 1664-9583 (Print-Version)

ISSN 1664-9591 (digitale Version)

11. Jahrgang Heft $2 / 2021$

https://doi.org/10.30820/1664-9583-2021-2

info@psychotherapie-wissenschaft.info

www.psychotherapie-wissenschaft.info

\section{Herausgeber}

Schweizer Charta für Psychotherapie in der Assoziation

Schweizer Psychotherapeutinnen und Psychotherapeuten

Geschäftsstelle ASP

Riedtlistr. 8

CH-8006 Zürich

Tel. +41432689300

www.psychotherapie.ch

\section{Redaktion}

Mara Foppoli, Lugano

Lea-Sophie Richter, Zürich

Mario Schlegel, Zürich

Peter Schulthess, Zürich

Hinweise für AutorInnen befinden sich auf der Homepage der Zeitschrift: www.psychotherapie-wissenschaft.info

\section{Verlag}

Psychosozial-Verlag

Walltorstr. 10

D-35390 Gießen

+49642196997826

info@psychosozial-verlag.de

www.psychosozial-verlag.de

\author{
Abo-Verwaltung \\ Psychosozial-Verlag \\ bestellung@psychosozial-verlag.de
}

\section{Bezugsgebühren}

Jahresabonnement 44,90€ (zzgl. Versand)

Einzelheft $24,90 €$ (zzgl. Versand)

Studierende erhalten gegen Nachweis $25 \%$ Rabatt.

Das Abonnement verlängert sich um jeweils ein Jahr, sofern nicht eine Abbestellung bis acht Wochen vor Ende des Bezugszeitraums erfolgt.

ASP-Mitglieder wenden sich wegen des Abonnements bitte direkt an die ASP.

\section{Anzeigen}

Anfragen zu Anzeigen bitte an den Verlag:

anzeigen@psychosozial-verlag.de

Es gelten die Preise der auf www.psychosozial-verlag.de einsehbaren Mediadaten.

ASP-Mitglieder wenden sich bitte direkt an

die ASP-Geschäftsstelle: asp@psychotherapie.ch

\section{Digitale Version}

Die Zeitschrift Psychotherapie-Wissenschaft ist auch online einsehbar: www.psychotherapie-wissenschaft.info

\section{(C) $(1) \Theta$}

Die Beiträge dieser Zeitschrift sind unter der Creative Commons Attribution-NonCommercial-NoDerivs 3.0 DE Lizenz lizensiert. Diese Lizenz erlaubt die private Nutzung und unveränderte Weitergabe, verbietet jedoch die Bearbeitung und kommerzielle Nutzung. Weitere Informationen finden Sie unter: creativecommons.org/licenses/by-nc-nd/3.0/de 


\title{
Psychothérapie en ligne et télé psychothérapie
}

\section{Sur le changement de décor lors de la pandémie de Covid 19}

\author{
Christiane Eichenberg, Paolo Raile, Stella Becher, Catherine Dapeci, Jessica Pacher, \\ Paul Jo Marc Rach, Birgitta Schiller, Eva Wimmer \& Lisa Winter
}

Psychotherapie-Wissenschaft 11 (2) $202181-82$

www.psychotherapie-wissenschaft.info

CC BY-NC-ND

https://doi.org/10.30820/1664-9583-2021-2-81

Mots clés : Covid-19, psychothérapie en ligne, télé psychothérapie, cadre, clinique ambulatoire psychothérapeutique

Contexte : Au printemps 2020, le coronavirus s'est répandu dans toute l'Europe. Le gouvernement fédéral autrichien a imposé un «lock down», c'est-à-dire l'obligation de ne sortir de ses quatre murs qu'à certaines occasions et de réduire massivement ses contacts sociaux. Il a donc fallu adapter le cadre psychothérapeutique, ce qui a également nécessité des changements dans les cliniques psychothérapeutiques ambulatoires, notamment celles de l'Université Sigmund Freud (SFU) à Vienne. Les psychothérapeutes ont changé de cadre et ont poursuivi les traitements sous la forme d'une psychothérapie en ligne ou d'une télé psychothérapie par Skype, Zoom ou téléphone et ont également commencé de nouvelles psychothérapies dans ce cadre. Question de recherche : évaluer les effets du changement de cadre induit par le lock down vers un traitement à distance et, après la fin du lock down, le retour au cadre traditionnel sur les thérapeutes et les clients de la clinique externe de SFU. Méthode : Une série d'enquêtes en ligne et d'entretiens avec des patients et des psychothérapeutes de la SFU Outpatient Clinic a été réalisée. Dans cet article, les textes des réponses ouvertes sont structurés au moyen d'une analyse de contenu résumée et leurs énoncés principaux sont présentés. Résultats : La flexibilité du lieu et la distance ont été décrites positivement par les thérapeutes et les patients ; d'un autre côté, ce dernier point en particulier a également conduit à des sentiments accrus de solitude et d'isolement chez les patients ; en outre, les patients étaient moins en mesure de s'impliquer émotionnellement dans la psychothérapie. Après être retournés dans le cadre du face-à-face, la majorité des participants ont déclaré qu'il était plus agréable, mais ont dit qu'il était maintenant plus difficile d'accepter l'augmentation du temps nécessaire pour le voyage, le temps d'attente et le retour à la maison par rapport au cadre en ligne. Conclusion : Globalement, on peut résumer que les résultats de l'enquête confirment que la psychothérapie en ligne ou la télé psychothérapie présente des avantages certains pour certains clients et qu'il peut donc être utile que les psychothérapeutes aient la possibilité de choisir le cadre avec leurs clients de manière flexible, même après la pandémie.

\section{Les Auteurs}

Christiane Eichenberg, professeure d'université, Dr. phil. habil., diplômée de psychologie, est psychanalyste et directrice de l'Institut de psychosomatique à la Faculté de médecine de l'Université privée Sigmund Freud à Vienne (SFU).

Paolo Raile, Dr., MSc, a étudié les sciences psychothérapeutiques à la SFU, le travail social à l'Université du Danube à Krems et termine actuellement deux doctorats à l'Université de Vienne en ethnologie européenne et à la SFU en science de la psychothérapie. Il est chercheur associé à la SFU, psychothérapeute, travailleur social, conseiller de vie et de société, fondateur et directeur de l'association Psychosocialis et de la société InContact $\mathrm{GmbH}$.

Stella Becher a terminé sa formation de formatrice agréée en prévention mentale et burnout en 2019. Elle est également conseillère psychosociale certifiée en gérontologie. Elle étudie actuellement la linguistique à l'Université de Vienne et les sciences psychothérapeutiques à la SFU, mention thérapie familiale systémique. Elle travaille comme coordinatrice de recherche à la SFU, Clinique ambulatoire pour adultes.

Catherine Dapeci, ancienne athlète de haut niveau en voile, est une psychologue et une psychothérapeute en herbe. Elle est éducatrice récréative spécialisée dans le sport, formatrice agréée par l'État en voile, coordinatrice de recherche à la SFU, Clinique ambulatoire pour adultes et participe à de nombreux projets de recherche.

Jessica Pacher fait des études en sciences psychothérapeutiques, mention thérapie familiale systémique à la SFU, travaille comme coordinatrice de recherche à la SFU, Clinique ambulatoire pour adultes et est rédactrice de la lettre d'information du Syndicat des étudiants autrichiens (Österreichische Hochschülerschaft ÖH) de la SFU.

Paul Jo Marc Rach, diplômé de licence en lettres, a étudié les sciences de l'éducation, axées sur l'éducation curative et le conseil à l'Université de Vienne, fait actuellement des études en sciences psychothérapeutiques à la SFU, mention thérapie comportementale, travaille comme coordinateur de recherche de 
la SFU, Clinique ambulatoire pour adultes et est l'interlocuteur des étudiants spécialisés dans une matière.

Birgitta Schiller, diplômée de maîtrise, universitaire assistante, a étudié les sciences psychothérapeutiques à la SFU, s'est spécialisée en psychologie individuelle, est une psychothérapeute agréée travaillant dans son propre cabinet et associée de recherche à l'Institut de recherche en psychothérapie qualitative et à l'Institut de psychosomatique de la SFU.

Eva Wimmer, diplômée de maîtrise de lettres, est une sociologue spécialisée dans les méthodes qualitatives/la recherche sociale interprétative et les études de genre. En tant qu'associée de recherche à l'Institut de recherche sur la psychothérapie qualitative de la SFU, elle mène actuellement des recherches, entre autres, sur le stress et les tensions du personnel médical causés par la Covid-19, et sur l'interface des sciences sociales et de la psychothérapie.

Lisa Winter, titulaire d'une double maîtrise, est psychothérapeute et spécialiste du théâtre, du cinéma et des médias, directrice adjointe de la clinique externe de psychothérapie, associée de recherche et chargée de cours en histoire culturelle, développement historique et intervention de crise à la SFU.

\section{Contact}

Dr. Paolo Raile

Sigmund Freud PrivatUniversität

Freudplatz 1/4009

1020 Wien

E-Mail : paolo.raile@sfu.ac.at 